\title{
Prevalence and characteristics of Australian women who use prayer or spiritual healing. A nationally representative cross-sectional study
}

RAO, ANGELA (RN, BN (Hons), PhD Candidate) ${ }^{a}$

HICKMAN, LOUISE. D. (RN, MPH, PhD)a

Director Postgraduate Nursing Studies

PHILLIPS, JANE. L. (PhD) ${ }^{\mathrm{b}}$

Professor of Palliative Care

SIBBRITT, DAVID (PhD)c

Professor of Epidemiology

${ }^{a}$ Faculty of Health, University of Technology Sydney, NSW, Australia

${ }^{b}$ Centre for Cardiovascular and Chronic Care, Sydney, NSW, Australia

${ }^{c}$ Australian Research Centre in Complementary and Integrative Medicine, Sydney, NSW, Australia

Corresponding author:

Angela Rao

Building 10, Level 6. PO Box 123 Ultimo NSW 2007 Australia

Ph (m) 0414530358; (w) 95142858

E-mail: Angela.Rao@uts.edu.au 
Objectives: To determine the prevalence and characteristics of users of prayer or spiritual healing among Australian women aged 31-36 years.

Design and setting: This cross-sectional study was conducted as a part of the Australian Longitudinal Study on Women's Health (ALSWH). The sample used in the current sub-study were participants from the 'young' cohort (1973-78) ( $n=8180)$ aged between 31- 36 years.

Main outcome measure: Use of prayer or spiritual healing

Results: Prayer or spiritual healing was used on a regular basis by $20 \%$ of women aged between 31 and 36 years in 2009. Women who had symptoms of chronic illnesses ( $p=0.001)$, women who had never smoked $(p=0.001)$ and women who used other forms of CAM $(p<0.001)$ were significantly more likely to use prayer or spiritual healing.

Conclusion: A significant proportion of women use prayer or spiritual healing on a regular basis. Further research is required to better understand their rationale for using prayer or spiritual healing and its perceived impact on health related outcomes and general wellbeing.

Keywords: Chronic illness, chronic disease, complementary and alternative medicine, integrative medicine, person centred care.

Word count: 3567

\section{Introduction}

Younger women are looking for alternative means to maintain their health, and/or manage their symptoms thereby decreasing reliance on conventional care (1-4). One way that younger women are seeking to address managing their symptoms is through prayer and/or spiritual healing $(5,6)$. Prayer or spiritual healing therapies are classified as complementary and alternative medicine (CAM), specifically, as mind body therapies, with some spiritual healing therapies such as Reiki also being categorised as energy healing therapies (4). 
Prayer is defined as the conscious willing intent of one or more persons for the well-being of self (personal prayer) or others (intercessory prayer) which may be initiated as a conscious activity or stimulated from the unconscious mind (7). Spiritual healing is defined as the "systematic, purposeful intervention by one or more persons aimed to help another person by focused intention to improve their condition" $(8, p .4)$. Spiritual healing can occur by use of non- contact healing at the bedside where hands are held a few inches above the body. Both intercessory prayer and spiritual healing may also occur over distance (termed remote intercessory prayer and distance healing) which may occur without prior physical contact or social interaction between the healer and the recipient (9).

Prayer has been used since antiquity and comprises a significant component of the belief systems of many cultures, including traditional or tribal based religious ideologies and the five major world religions (Christianity, Judaism, Buddhism, Islam and Hinduism) (10). Spiritual healing remains an integral part of traditional health care in many non-Western countries $(9,11)$.

Complementary and alternative medicine can be broadly defined as a group of medical and health related practices, disciplines or products that are not considered to be within the scope of conventional medicine (4). CAM also includes therapies that are considered a part of the traditional practices of Non-Western countries, such as Traditional Chinese Medicine or Ayurveda (12) Previous literature has indicated that CAM practices generally are found to be used more among women compared to men, particularly in regards to use of mind-body therapies such as prayer or spiritual healing (12). Use of prayer or spiritual healing has been correlated with improved health outcomes for adults living in the community. For example, in a sample of healthy adults (mean age 34 years; SD 8) personal religious prayer has been associated with reduced respiratory rate, improved regularity similar to controlled breathing exercises, increased heart rate variability and improved synchronisation (13). Spirituality and faith is also perceived to be beneficial as a means of guidance, support, strength, confidence and protection among pregnant women (6).

A Cochrane review concluded that whilst the evidence for intercessory prayer is inconclusive, given the lack of adverse effects, advised that current practices in relation to the use of intercessory prayer should be maintained $(14,15)$. Similarly, three systematic reviews determining the effectiveness of spiritual healing were inconclusive and stated that the area warranted further research including methodologically strong studies that assess expectation and belief (16-18). There is evidence to suggest that use of prayer or spiritual healing is increasing, with $42 \%$ of American cancer survivors utilising faith or spiritual healing, and $61 \%$ utilising prayer or spiritual practice (5). Therapeutic Touch, an energy healing modality that incorporates laying of hands, was also used by $3 \%$ of this sample (5). Energy healing modalities are used in over 50 teaching hospitals in the United States, not including its use in private practice (19). 


\section{Aim}

This study aimed to determine the prevalence of use; demographic, health status and health service utilisation factors associated with the use of prayer or spiritual healing among Australian women aged between $31-36$ years (in 2009) using a nationally representative sample $(n=18,145)$, and in doing so provide important information of relevance to women who use prayer or spiritual healing, health care practitioners and policy makers (20). Characteristics of use of prayer or spiritual healing among women aged 59-64 years has been explored in a separate paper (21).

\section{Methods}

\section{Sample:}

The Australian Longitudinal Study on Women's Health (ALSWH) is a population-based study that aims to examine the physical and emotional health, socio-demographic factors, health service utilisation, health behaviours and risk factors, and the significant life events of the women over a period of twenty years (22). Women involved in the study were recruited using random selection into one "young" cohort (1973-78), which was a nationally representative sample of the population (23). Initial surveys in 1996 included 14,779 respondents in the young cohort, however only the most recent survey (2009) was used in the analysis (20).

Eligibility criteria included women that were registered on the Australian Medicare database, which has registered details of the names and addresses of permanent residents and citizens including refugee and migrant women $(22,24)$. Of selected participants, the response rate was $62 \%$ from the $1973-78$ cohort in 2009 , with the main reason for nonresponse due to inability to contact women after all possible means were used (25).

Response rates cannot be precisely specified due to uncertainties regarding accuracy of the Medicare database (25). Informed consent was obtained by means of a returned signed consent form attached to the initial invitation to participate and questionnaire (22). Women with a tertiary education were overrepresented whilst some migrant groups of women were underrepresented introducing response bias (22). Ethics approval for use of the dataset was gained from the Human Research Ethics Committees at the University of Queensland, the University of Newcastle and the University of Technology Sydney (23).

\section{Measures of demographic characteristics}

To determine the area of residence as urban, rural or remote, current geographical postcode was used. Information regarding marital status, age (in years), ability to manage on available income, level of education, smoking status, alcohol use, and health insurance for hospital cover or ancillary services was obtained. 


\section{Measures of health status}

Measures of health status included frequency of experiencing health symptoms in the past 12 months such as difficulty breathing, stiff or painful joints, back pain constipation, difficulty sleeping, intense anxiety such as panic attacks, other mental health problems or palpitations. Women were asked about any diagnosed conditions within the past three years including hypertension, asthma, bronchitis, skin cancer or other cancer. The SF-36 Quality of Life Questionnaire was used to assess physical and mental health related quality of life (26). It contained eight domains (physical functioning, role physical, bodily pain, general health, vitality, social functioning, role-emotional and mental health), with higher scores reflecting greater health (26).

\section{Outcome measure}

Women from the 1973-78 cohort (aged 31 to 36 years in 2009) were asked whether they had used prayer or spiritual healing in the last 12 months, and were given the response options of 'never', 'rarely', 'sometimes' or 'often'.

\section{Statistical analysis}

Chi-squared tests were used to determine the associations between categorical demographic and health status factors and the use of prayer or spiritual healing. Differences in mean scores between continuous demographic and health status factors and the use of prayer or spiritual healing were tested using one way analysis of variance (ANOVA). For each cohort, statistically significant $(p<0.25)$ demographic and health status factors were entered into a logistic regression model and then a stepwise backward elimination process using a likelihood ratio test was used to determine the model of best fit (27). Given the large sample size, statistical significance was set at a $p$-value of $p<0.005$. Statistical analyses were all completed using the STATA statistical program.

\section{Results}

A total of 8,180 (99.8\%) women from this sub-study (1973-78) cohort answered the question regarding the use of prayer or spiritual healing. Prayer or spiritual healing was used often by $9 \%(n=766)$, used sometimes by $11 \%(n=883)$, used rarely by $11 \%(n=887)$ and never used by $69 \%(n=5,644)$.

Demographic characteristics of women aged 31-36 years (in 2009) who use prayer or spiritual healing are described in Table 1. Women who used prayer or spiritual healing were significantly more likely to have never married $(p<0.001)$, obtained a university degree $(p<0.001)$, never smoked $(p<0.0001)$, and less likely to have hospital cover $(p<0.001)$.

Table 1: The association between demographic characteristics and use of prayer or spiritual healing by women aged 31-36 years in 2009.

\section{Characteristics}

Use of prayer and spiritual healing

\begin{tabular}{|c|c|c|c|c|}
\hline $\begin{array}{c}\text { Never } \\
(n=5644)\end{array}$ & $\begin{array}{c}\text { Rarely } \\
(n=887)\end{array}$ & $\begin{array}{c}\text { Sometimes } \\
(n=883)\end{array}$ & $\begin{array}{c}\text { Often } \\
(n=766)\end{array}$ & p value \\
\hline
\end{tabular}




\begin{tabular}{|c|c|c|c|c|c|}
\hline $\begin{array}{l}\text { Urban } \\
\text { Rural/Remote }\end{array}$ & $\begin{array}{l}\% \\
60 \\
40\end{array}$ & $\begin{array}{l}\% \\
61 \\
39\end{array}$ & $\begin{array}{l}\% \\
60 \\
40\end{array}$ & $\begin{array}{l}\% \\
60 \\
40\end{array}$ & 0.856 \\
\hline $\begin{array}{ll}\text { Marital status } & \text { Never married } \\
& \text { Married/defacto } \\
& \text { Sep./Div./Wid. }\end{array}$ & $\begin{array}{c}16 \\
79 \\
5\end{array}$ & $\begin{array}{l}18 \\
76 \\
6\end{array}$ & $\begin{array}{l}19 \\
74 \\
7\end{array}$ & $\begin{array}{c}20 \\
74 \\
6\end{array}$ & 0.001 \\
\hline $\begin{array}{l}\text { Impossible/difficult } \\
\text { Difficult sometimes } \\
\text { Not too bad/easy }\end{array}$ & $\begin{array}{l}11 \\
28 \\
61\end{array}$ & $\begin{array}{l}14 \\
29 \\
57\end{array}$ & $\begin{array}{l}13 \\
30 \\
57\end{array}$ & $\begin{array}{l}15 \\
28 \\
57\end{array}$ & 0.018 \\
\hline $\begin{array}{l}\text { School only } \\
\text { Trade/Certificate } \\
\text { University degree }\end{array}$ & $\begin{array}{l}23 \\
27 \\
50\end{array}$ & $\begin{array}{l}18 \\
27 \\
55\end{array}$ & $\begin{array}{l}17 \\
26 \\
57\end{array}$ & $\begin{array}{l}18 \\
27 \\
55\end{array}$ & $<0.001$ \\
\hline $\begin{array}{l}\text { Never smoked } \\
\text { Ex-smoker } \\
\text { Current smoker }\end{array}$ & $\begin{array}{l}59 \\
26 \\
15\end{array}$ & $\begin{array}{l}58 \\
26 \\
16\end{array}$ & $\begin{array}{l}60 \\
26 \\
14\end{array}$ & $\begin{array}{l}67 \\
23 \\
10\end{array}$ & 0.001 \\
\hline $\begin{array}{l}\text { No/low risk } \\
\text { Risky/high risk }\end{array}$ & $\begin{array}{c}95 \\
5\end{array}$ & $\begin{array}{c}96 \\
4\end{array}$ & $\begin{array}{c}96 \\
4\end{array}$ & $\begin{array}{c}96 \\
4\end{array}$ & 0.590 \\
\hline $\begin{array}{ll}\text { Private hospital } & \text { Yes } \\
\text { cover } & \text { No } \\
\end{array}$ & $\begin{array}{l}63 \\
37 \\
\end{array}$ & $\begin{array}{l}66 \\
34 \\
\end{array}$ & $\begin{array}{l}67 \\
33 \\
\end{array}$ & $\begin{array}{l}57 \\
43\end{array}$ & $<0.001$ \\
\hline $\begin{array}{ll}\text { Private ancillary } & \text { Yes } \\
\text { cover } & \text { No }\end{array}$ & $\begin{array}{l}61 \\
39 \\
\end{array}$ & $\begin{array}{l}64 \\
36 \\
\end{array}$ & $\begin{array}{l}62 \\
38 \\
\end{array}$ & $\begin{array}{l}58 \\
42 \\
\end{array}$ & 0.090 \\
\hline Age & $\begin{array}{c}\begin{array}{c}\text { Mean } \\
\text { (SD) }\end{array} \\
33.7(1.5)\end{array}$ & $\begin{array}{c}\begin{array}{c}\text { Mean } \\
\text { (SD) }\end{array} \\
33.6(1.5)\end{array}$ & $\begin{array}{l}\text { Mean (SD) } \\
33.6(1.5)\end{array}$ & $\begin{array}{c}\begin{array}{c}\text { Mean } \\
\text { (SD) }\end{array} \\
33.7(1.5)\end{array}$ & $\begin{array}{l}\text { p value } \\
0.210\end{array}$ \\
\hline
\end{tabular}

Symptoms of chronic illness and use of prayer or spiritual healing

In comparison to women who never or rarely experienced any of these symptoms, women that used prayer or spiritual healing were significantly more likely to have sometimes or often experienced difficulty breathing $(p<0.001)$; back pain $(p<0.001)$; stiff or painful joints $(p<0.001)$; constipation $(p<0.001)$; difficulty sleeping $(p<0.001)$; depression $(p<0.001)$; intense anxiety or panic attacks $(p<0.001)$; other mental health problems $(p<0.001)$; and/or palpitations $(p<0.001)$ (See Table 2$)$.

Table 2: The association between symptoms of chronic illness and use of prayer or spiritual healing by women aged 31-36 years in 2009.

\begin{tabular}{|c|c|c|c|c|c|c|}
\hline \multirow{2}{*}{\multicolumn{2}{|c|}{ Health Symptoms }} & \multicolumn{5}{|c|}{ Use of prayer or spiritual healing } \\
\hline & & $\begin{array}{c}\text { never } \\
(n=5644)\end{array}$ & $\begin{array}{c}\text { rarely } \\
(n=887)\end{array}$ & $\begin{array}{c}\text { sometimes } \\
(n=883)\end{array}$ & $\begin{array}{c}\text { often } \\
(n=766)\end{array}$ & P value \\
\hline \multirow{3}{*}{$\begin{array}{l}\text { Difficulty } \\
\text { breathing }\end{array}$} & & $\%$ & $\%$ & $\%$ & $\%$ & \multirow{3}{*}{0.001} \\
\hline & Never/Rarely & 93 & 93 & 90 & 90 & \\
\hline & Sometimes/Often & 7 & 7 & 10 & 10 & \\
\hline Stiff or painful & Never/Rarely & 77 & 74 & 73 & 71 & $<0.001$ \\
\hline
\end{tabular}




\begin{tabular}{|ll|c|c|c|c|c|}
\hline joints & Sometimes/Often & 23 & 26 & 27 & 29 & \\
\hline Back pain & Never/Rarely & 57 & 54 & 50 & 50 & $<0.001$ \\
& Sometimes/Often & 43 & 46 & 50 & 50 & \\
\hline Constipation & Never/ Rarely & 83 & 79 & 77 & 79 & $<0.001$ \\
& Sometimes/Often & 17 & 21 & 23 & 21 & \\
\hline Difficulty & Never/Rarely & 67 & 63 & 59 & 60 & $<0.001$ \\
sleeping & Sometimes/Often & 33 & 37 & 41 & 40 & \\
\hline Depression & Never/Rarely & 82 & 74 & 74 & 73 & $<0.001$ \\
& Sometimes/Often & 18 & 26 & 26 & 27 & \\
\hline Panic attacks/ & Never/Rarely & 92 & 87 & 88 & 86 & $<0.001$ \\
intense anxiety & Sometimes/Often & 8 & 13 & 12 & 14 & \\
\hline Other mental & Never/Rarely & 97 & 95 & 95 & 93 & $<0.001$ \\
health problems & Sometimes/Often & 3 & 5 & 5 & 7 & \\
\hline Palpitations & Never/Rarely & 89 & 86 & 84 & 82 & $<0.001$ \\
& Sometimes/Often & 11 & 14 & 16 & 18 & \\
\hline
\end{tabular}

Diagnosed chronic illnesses and use of prayer or spiritual healing

There were no significant associations between diagnosed conditions and use of prayer or spiritual healing (see Table 3).

Table 3: The association between diagnosed chronic illnesses and use of prayer or spiritual healing by women aged 31-36 years in 2009.

\begin{tabular}{|ll|c|c|c|c|c|}
\hline \multirow{2}{*}{ Diagnosed condition } & & \multicolumn{5}{|c|}{ Use of prayer or spiritual healing } \\
\cline { 3 - 7 } & & $\begin{array}{c}\text { Never } \\
(\mathrm{n}=5644)\end{array}$ & $\begin{array}{c}\text { Rarely } \\
(\mathrm{n}=887)\end{array}$ & $\begin{array}{c}\text { Sometimes } \\
(\mathrm{n}=883)\end{array}$ & $\begin{array}{c}\text { Often } \\
(\mathrm{n}=766)\end{array}$ & p value \\
\hline \multirow{3}{*}{ Hypertension } & & $\%$ & $\%$ & $\%$ & $\%$ & \\
& No & 96 & 95 & 96 & 95 & 0.685 \\
\hline Asthma & Yes & 4 & 5 & 4 & 5 & \\
\hline Bronchitis & No & 90 & 89 & 89 & 90 & 0.823 \\
& Yes & 10 & 11 & 11 & 10 & \\
\hline Skin cancer & No & 94 & 94 & 93 & 93 & 0.373 \\
& Yes & 6 & 6 & 7 & 7 & \\
\hline Other cancer & No & 98 & 97 & 97 & 97 & 0.187 \\
& Yes & 2 & 3 & 3 & 3 & \\
\hline & No & 99 & 99 & 99 & 99 & 0.384 \\
\hline
\end{tabular}

\section{Health Status}

Women that used prayer or spiritual healing sometimes or often had significantly more optimistic Life Orientation Test (LOT-R) scores than women who used prayer or spiritual healing never or rarely $(p<0.001)$ (see Table 4$)$. In terms of health-related quality of life, women who used prayer or spiritual healing sometimes or often had significantly poorer 
mental and physical health (in 7 of the 8 the SF-36 domains), compared to women who used prayer or spiritual healing never or rarely $(p \leq 0.001)$. General health was also poorer in women who used spiritual healing sometimes or often, however this was not significant $(p=0.009)$. Clinically significant differences in SF-36 domains (defined as $\geq 3$ points) were found for role physical (3.2 points), social functioning (4 points), vitality (3.1 points), role emotional (8.2 points) and mental health (3.4 points) (26).

Table 4: The association between health status and use of prayer or spiritual healing by women from the (1973-78) cohort.

\begin{tabular}{|c|c|c|c|c|c|c|}
\hline \multirow{2}{*}{ Health status } & & \multicolumn{5}{|c|}{ Use of prayer or spiritual healing } \\
\hline & & $\begin{array}{l}\text { Never } \\
(n=5644)\end{array}$ & $\begin{array}{l}\text { Rarely } \\
(\mathrm{n}=887)\end{array}$ & $\begin{array}{l}\text { Sometimes } \\
(\mathrm{n}=883)\end{array}$ & $\begin{array}{l}\text { Often } \\
(n=766)\end{array}$ & p value \\
\hline \multirow{5}{*}{$\begin{array}{l}\text { BMI } \\
\text { classification }\end{array}$} & & $\%$ & $\%$ & $\%$ & $\%$ & \\
\hline & underweight & 2 & 3 & 3 & 4 & 0.009 \\
\hline & healthy weight & 51 & 56 & 54 & 55 & \\
\hline & overweight & 26 & 23 & 26 & 23 & \\
\hline & obese & 21 & 18 & 17 & 18 & \\
\hline \multirow[t]{4}{*}{ METS } & none & 13 & 11 & 13 & 15 & 0.008 \\
\hline & low & 26 & 26 & 28 & 28 & \\
\hline & moderate & 10 & 10 & 10 & 11 & \\
\hline & high & 51 & 53 & 49 & 46 & \\
\hline \multirow{2}{*}{\multicolumn{2}{|c|}{ Life orientation test }} & Mean (SD) & Mean (SD) & Mean (SD) & Mean (SD) & p value \\
\hline & & $15.6(4.3)$ & $15.8(4.1)$ & $16.2(4.3)$ & $16.4(4.4)$ & $<0.001$ \\
\hline \multicolumn{2}{|c|}{ SF-36 physical function } & $90.6(15.8)$ & $90.4(14.7)$ & $88.3(17.7)$ & $88.8(17.3)$ & 0.001 \\
\hline \multicolumn{2}{|c|}{ SF-36 role physical } & $82.5(32.3)$ & $79.3(33.3)$ & 76.1 (35.9) & $77.2(35.4)$ & $<0.001$ \\
\hline \multicolumn{2}{|c|}{ SF- 36 bodily pain } & $75.1(21.0)$ & $73.3(19.9)$ & $71.2(21.5)$ & $71.4(22.4)$ & $<0.001$ \\
\hline \multicolumn{2}{|c|}{ SF- 36 general health } & $74.2(18.7)$ & $71.7(18.8)$ & $73.1(18.8)$ & $72.9(20.3)$ & 0.009 \\
\hline \multicolumn{2}{|c|}{ SF- 36 social functioning } & $84.3(21.3)$ & $79.3(23.0)$ & $79.2(23.3)$ & $78.9(24.1)$ & $<0.001$ \\
\hline \multicolumn{2}{|l|}{ SF- 36 vitality } & $54.5(20.0)$ & $51.4(20.0)$ & $52.4(19.7)$ & $51.8(20.4)$ & $<0.001$ \\
\hline \multicolumn{2}{|c|}{ SF-36 role emotional } & $81.6(33.4)$ & $73.4(37.5)$ & $74.7(37.4)$ & $73.9(38.6)$ & $<0.001$ \\
\hline \multicolumn{2}{|c|}{ SF-36 mental health } & $73.3(16.3)$ & $69.9(17.1)$ & $70.7(16.4)$ & $71.7(17.2)$ & $<0.001$ \\
\hline
\end{tabular}

\section{Complementary Therapies}

Women who used vitamins or minerals sometimes or often were significantly more likely to use prayer or spiritual healing compared with women who used vitamins or minerals never or rarely $(p<0.001$ ) (see Table 5$)$. Compared with women who used yoga or meditation never or rarely, women who used yoga or meditation sometimes or often were significantly more likely to use prayer or spiritual healing $(p<0.001)$. Women who sometimes or often used herbal medicines were significantly more likely to use prayer or spiritual healing compared with women who never or rarely used herbal medicines $(p<0.001)$. Use of prayer 
or spiritual healing was significantly more likely in women who used aromatherapy sometimes or often compared with women who used aromatherapy never or rarely $(p<0.001)$. In comparison with women who used Chinese medicines never or rarely, women who sometimes or often used Chinese medicines were significantly more likely to use prayer or spiritual healing $(p<0.001)$. Women who sometimes or often used another alternative therapy were significantly more likely to use prayer or spiritual healing compared with women who used another alternative therapy never or rarely $(p<0.001)$.

Table 5: The association between use of complementary therapies and use of prayer or spiritual healing by women aged 31-36 years in 2009.

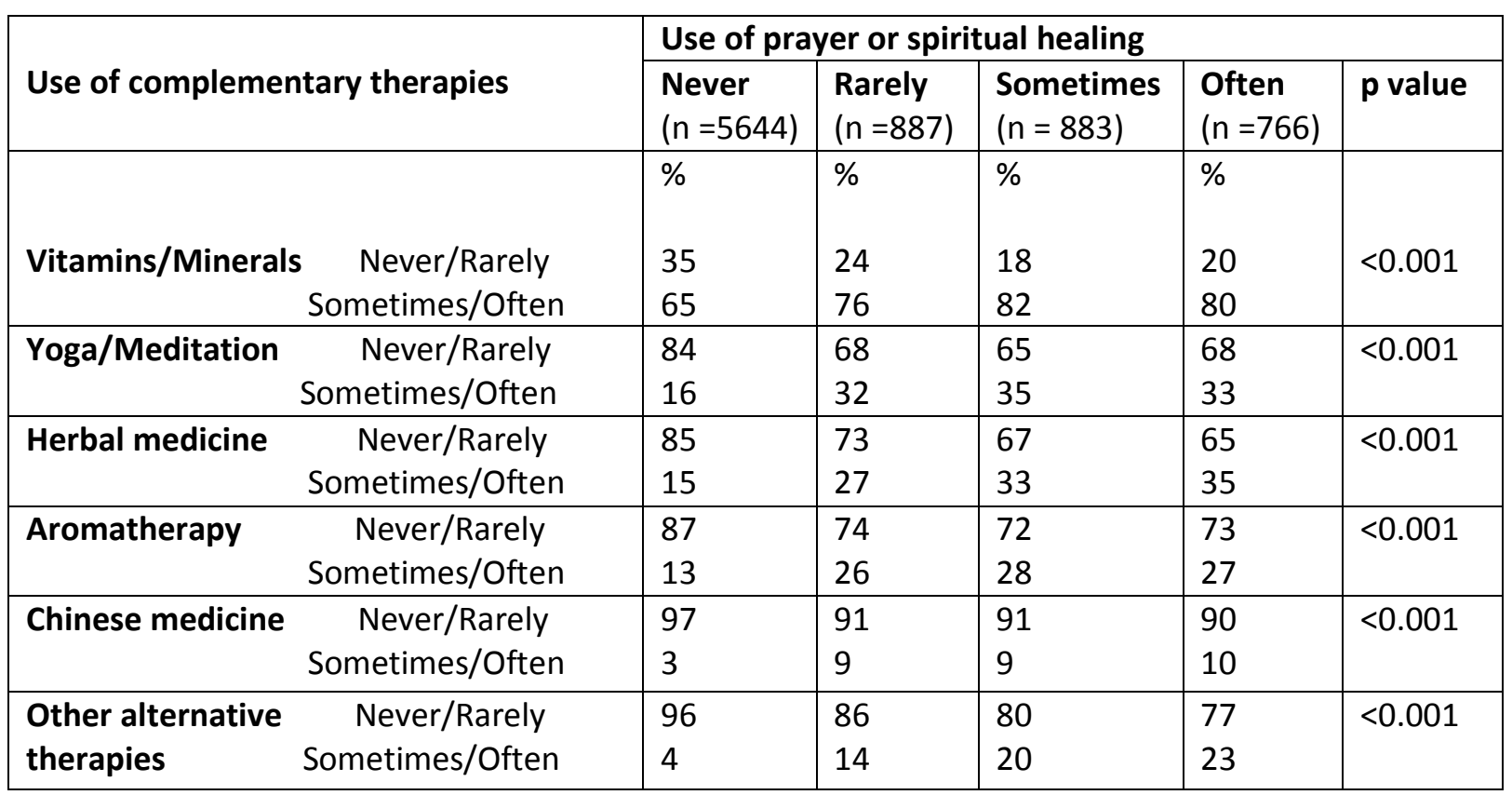

\section{Logistic Regression Modelling}

Compared to women who have never smoked, ex-smokers are 0.78 ( $95 \% \mathrm{Cl}: 0.67,0.90)$ times less likely to use prayer or spiritual healing whilst current smokers were $0.70(95 \% \mathrm{Cl}$ : $0.58,0.85$ ) times less likely to use prayer or spiritual healing (see Table 6).

Women that report having palpitations sometimes or often are $1.32(95 \% \mathrm{Cl}: 1.11,1.58)$ times more likely to use prayer or spiritual healing than women that reported palpitations never or rarely. Women who consult a counsellor or other mental health worker were 0.78 $(95 \% \mathrm{Cl}: 0.64,0.89)$ times less likely to use prayer or spiritual healing than women who did not consult a counsellor or other mental health worker. Compared to women who did not consult an acupuncturist, women who consulted an acupuncturist were 1.45 (95\% Cl: 1.16, 1.80) times more likely to use prayer or spiritual healing.

Women who used vitamins or minerals sometimes or often were $1.47(95 \% \mathrm{Cl}: 1.26,1.71)$ times more likely to use prayer or spiritual healing than women who used vitamins or minerals never or rarely. Similarly, the use of herbal medicines sometimes or often by women was associated with a $1.34(95 \% \mathrm{Cl}: 1.15,1.57)$ times greater likelihood of using 
prayer or spiritual healing than women who use herbal medicines never or rarely.

Compared to women who used yoga or meditation never or rarely, women who reported using yoga or meditation sometimes or often were $1.60(95 \% \mathrm{Cl}: 1.38,1.84)$ times more likely to use prayer or spiritual healing. Compared to women who used aromatherapy never or rarely, women who used aromatherapy sometimes or often were 1.33 (95 \% Cl: 1.14, 1.56) times more likely to use prayer or spiritual healing. Women who used other alternative therapies sometimes or often were 2.77 (95\% Cl: $2.28,3.38$ ) times more likely to use prayer or spiritual healing than women who did not use other alternative therapies.

For every 1 point increase on the life orientation test (i.e. more positive outlook on life) there was a corresponding $4 \%$ increase in the likelihood that women will use prayer or spiritual healing (OR=1.04; 95\% Cl: 1.02, 1.05). Similarly, for every 1 point increase in the SF36 social functioning domain (i.e. better social functioning) there was a corresponding $1 \%$ decrease in the likelihood that women will use prayer or spiritual healing $(\mathrm{OR}=0.99 ; 95 \% \mathrm{Cl}$ : $0.98,1.00)$.

Table 6: Multiple logistic regression model for predicting use of prayer or spiritual healing compared for women aged 31-36 years in 2009.

\begin{tabular}{|ll|c|c|c|}
\hline Factor & & Odds Ratio & 95\% C.I. & p-value \\
\hline \multirow{2}{*}{ Smoking Status } & Non-smoker & 1.00 & - & \\
& Ex-smoker & 0.78 & $0.67,0.90$ & 0.001 \\
& Current smoker & 0.70 & $0.58,0.85$ & 0.001 \\
\hline Palpitations & Never/ rarely & 1.00 & - & \\
& Sometimes/ often & 1.32 & $1.11,1.58$ & 0.002 \\
\hline Consulted a Counsellor or & No & 1.00 & - & \\
other mental health worker & Yes & 0.76 & $0.64,0.89$ & 0.001 \\
\hline Consulted an & No & 1.00 & - & \\
Acupuncturist & Yes & 1.45 & $1.16,1.80$ & 0.001 \\
\hline Vitamins/ & Never/Rarely & 1.00 & - & \\
minerals & Sometimes/Often & 1.47 & $1.26,1.71$ & 0.001 \\
\hline Yoga/ meditation & Never/Rarely & 1.00 & - & \\
& Sometimes/Often & 1.60 & $1.38,1.84$ & 0.001 \\
\hline Herbal remedy & Never/Rarely & 1.00 & - & \\
& Sometimes/Often & 1.34 & $1.15,1.57$ & 0.001 \\
\hline Aromatherapy & Never/Rarely & 1.00 & - & \\
& Sometimes/Often & 1.33 & $1.14,1.56$ & 0.001 \\
\hline Other alternative & Never/Rarely & 1.00 & - & \\
therapy & Sometimes/Often & 2.77 & $2.28,3.38$ & 0.001 \\
\hline Life orientation test & & 1.04 & $1.02,1.05$ & 0.001 \\
& & & & \\
\hline Sf-36 social functioning & & 0.99 & $0.99,1.00$ & 0.001 \\
& & & & \\
\hline
\end{tabular}




\section{Discussion}

This is one of the few studies examining the use of prayer or spiritual healing among a nationally-representative sample of women aged between 31-36 years. A number of important issues have been highlighted regarding prayer or spiritual healing use, including its use for chronic illness symptom management and as a preventative health practice.

In 2009, 20\% of Australian women aged 31-36 years reported regular use of prayer or spiritual healing. This was lower than the $41 \%$ of adults aged between $30-39$ years, and $53 \%$ of women overall who use prayer for health reasons in the American setting (28). This may be due to the inclusion of males in the sample and may reflect higher trends of prayer use within the American population (28). Prayer or spiritual healing is used at a slightly lower rate among women aged 31-36 years compared to the 'mid cohort' of women aged 59-64 years, which may reflect the lower rates of chronicity and comorbidity among younger women (21). Women managing symptoms of chronic illnesses were significantly more likely to use prayer or spiritual healing, which is consistent with the findings of previous data where prayer or spiritual practices were used by $38.2 \%$ of adults between $25-75$ (mean age 45 years) with both physical and mental disorders (29). It may be that prayer or spiritual healing practices are being used to mediate the impact of psychosocial stressors and higher levels of psychological distress associated with chronic illness symptoms in younger women $(30,31)$.

A review of the prayer practices of hospitalised patients demonstrated improved coping, and identified prayer as a mediator between religious faith and well-being (32). Devotional prayer to a supportive God was associated with improved optimism, functioning and wellbeing whilst petitionary prayer was associated with increased distress and poorer function, particularly where previous religious faith was absent (32). Other findings also indicate that women who use prayer or spiritual healing have increased levels of distress, decline in health or functional status, and may be using these modalities as a positive coping strategy, as a hope for healing, or to reduce side effects such as pain $(33,34)$. Prayer or spiritual healing may also be used to manage stress or enhance coping by facilitating a broader, more philosophical perspective through connection with a divine source or something greater than one's self, and lead to a sense of empowerment (34).

Openness, perceived social support and positive appraisal from friends has been significantly associated with an increased use of mind body therapies, particularly energy therapies (29). This may suggest that psychological and spiritual support obtained by attending a prayer group, or by engaging in healing practices may assist in coping with the lived experience of chronic illness, for example, shared communication of pain, a sense of belonging or connection to a community, and the reinforcement of a shared belief system (35). This is of particular relevance given that women with poorer mental or physical health, were more likely to regularly use prayer or spiritual healing. 
Prayer or spiritual healing was significantly more likely to be used among women who had palpitations. This finding is consistent with previous literature (36), and is an example of the potential for prayer or spiritual healing to facilitate physiological benefit by inducing a relaxation response and decreasing hypothalamic adrenal pituitary axis activation $(37,38)$.

Use of prayer or spiritual healing was significantly more likely to be used among women with positive health seeking behaviours or lifestyle patterns (non-smokers) which is supported by findings of a previous review that positively associates increased religiosity and spirituality with preventative health practices such as reduced smoking, increased physical activity, healthy dietary behaviours and reduced tendency to engage in risky sexual activity (39). Another example of preventative health practices among women who use prayer or spiritual healing is their increased likelihood of utilising other complementary therapies which is consistent with previous data (28), and is also similar to the practices of Australian women aged 59-64 years (21).

As a part of preventative practices women may be exploring prayer or spiritual healing as an expression of the connection between mind, body and spirit. This is supported by psychoneuroimmunology research and is also consistent with Eastern traditions that have long acknowledged the roles of consciousness and the subconscious mind in the management of health, as evidenced in the conceptual framework of modalities such as Indian homeopathy, acupuncture and Traditional Chinese Medicine (40). An understanding of subtle energy is also a common denominator in the framework of these and other complementary therapies such as craniosacral osteopathy, kinesiology, bioenergy therapies and Alexander technique, which may account for why women who use prayer or spiritual healing may be using other forms of $\operatorname{CAM}(28,41)$.

There are a number of limitations to this research that need to be considered when interpreting the findings. This analysis did not specify the frequency or duration or types of prayer (intercessory or personal) or spiritual healing or conditions in which prayer or spiritual healing were used. Further, the use of a self-report method of data collection introduces recall bias (20). The analysis differentiated between symptoms and diagnosed conditions however findings were not confirmed with medical records. Differences in use among minority population groups were not assessed, which restricted comparisons made in previous studies (28). Cross sectional analyses are used to determine associations and assumptions can only be made regarding causal relationships (28). These limitations are minor compared to benefits gained from analysing a large nationally-representative sample of women (20). The response rate was $62 \%$, which may introduce a participation bias, and given the exclusion of men from the dataset, decreases the generalisability of findings to the population at large. 


\section{Implications for practice}

Western society is beginning to acknowledge the role of preventative health practices in the achievement and maintenance of health. The contribution of research on mind body practices such as prayer or spiritual healing is that it highlights a gap in conventional health care practices and delivery and encourages adults in the community to acknowledge their responsibility for the underlying factors that contribute to 'dis-ease' including lifestyle choices and disharmony between the individual and their environment (42).

It is important to emphasise that health care providers do not routinely assess for the use of mind-body practices such as prayer or spiritual healing. Adults in the community who engage in these practices may wish to engage in a dialogue with health care providers by initiating conversation regarding use, any benefits they may be receiving and how these therapies may assist with the optimisation of health outcomes. Health care providers also need to be aware that their patients may be using prayer or spiritual healing as adjunct therapies to conventional care and it is likely that these therapies are being used for symptom management of chronic illnesses, and as preventative health practices. A holistic patient centred approach needs to be supported at an organisational level in order to affect change among health care professionals that assist patients' enhanced management of their chronic illnesses (43). Education strategies within health care settings such as an assessment of the spiritual needs and prayer or healing preferences of patients should be encouraged (32).

\section{Conclusion}

A significant proportion of Australian women are using prayer or spiritual healing. Health care provider knowledge can be enhanced by understanding that women are using prayer or spiritual healing as non-pharmacological adjunct therapies, and as preventative health practices. Given that women may be using prayer or spiritual healing modalities as an adjunctive symptom management strategy, it is in the interest of policy makers and health care providers to respect the spiritual and religious preferences of these women and to provide an environment that promotes spiritual expression within health care settings. Further research is required to better understand their rationale for using prayer or spiritual healing and it's perceived impact on health related outcomes and general well-being.

\section{Conflict of interest statement}

None declared. 


\section{References}

1. Armstrong AR, Thiébaut SP, Brown L, Nepal B. Australian adults use complementary and alternative medicine in the treatment of chronic illness: a national study. Australian And New Zealand Journal Of Public Health. 2011;35(4):384-90.

2. World Health Organisation. Global Status Report on Non Communicable Diseases. World Health Organisation, 2014.

3. Anderson JG, Taylor AG. Use of complementary therapies by individuals with or at risk for cardiovascular disease: results of the 2007 National Health Interview Survey. The Journal of cardiovascular nursing. 2012;27(2):96-102.

4. National Centre for Complementary and Alternative Medicine. Third Strategic Plan 20112015. Exploring the science of complementary and alternative medicine. United States: National Institute of Health, 2011 2011. Report No.: Contract No.: 11-7643.

5. Gansler T, Kaw C, Crammer C, Smith T. A population-based study of prevalence of complementary methods use by cancer survivors. Cancer. 2008;113(5):1048-57.

6. Jesse DE, Schoneboom C, Blanchard A. The Effect of Faith or Spirituality in Pregnancy: A Content Analysis. Journal of Holistic Nursing. 2007;25(3):151-8.

7. Dossey L. Healing Words: The Power of Prayer and The Practice of Medicine. New York: HarperCollins; 1993.

8. Benor DJ. Spiritual healing: Scientific validation of a healing revolution: Wholistic Healing Pubns; 2001. 597 p.

9. Leder D. "Spooky actions at a distance": physics, psi, and distant healing. Journal Of Alternative And Complementary Medicine (New York, NY). 2005;11(5):923-30.

10. Brown LB. The human side of prayer: The psychology of praying. . Birmingham: Religious Education Press; 1994.

11. Walach H, Boesch H, Lewith G, Naumann J, Schwarzer B, Falk S, et al. Effectiveness of Distant Healing for Patients with Chronic Fatigue Syndrome: A Randomised Controlled Partially Blinded Trial (EUHEALS). Psychotherapy and Psychosomatics. 2008;77(3):158-66.

12. Barnes PM, Powell-Griner E, McFann K, Nahin RL. Complementary and alternative medicine use among adults: United States, 2002. Seminars in Integrative Medicine. 2004;2(2):54-71.

13. Bernardi L, Sleight $P$, Bandinelli $G$, Cencetti $S$, et al. Effect of rosary prayer and yoga mantras on autonomic cardiovascular rhythms: Comparative study. British Medical Journal.

2001;323(7327):1446-9.

14. Roberts L, Ahmed I, Hall S, Davison A. Intercessory prayer for the alleviation of ill health. Cochrane Database Syst Rev. 2009;1.

15. Hodge DR. A Systematic Review of the Empirical Literature on Intercessory Prayer. Research on Social Work Practice. 2007;17(2):174-87.

16. Abbot NC. Healing as a therapy for human disease: a systematic review. The Journal of Alternative and Complementary Medicine. 2000;6(2):159-69.

17. Astin JAP, Harkness EB, Ernst EMDP. The Efficacy of "Distant Healing": A Systematic Review of Randomized Trials. Annals of Internal Medicine. 2000;132(11):903-10.

18. Crawford C, Sparber A, Jonas W. A systematic review of the quality of research on hands-on and distance healing: clinical and laboratory studies. Alternative Therapies in Health and Medicine. 2003;9(3):96-104.

19. DiNucci EM. Energy healing: a complementary treatment for orthopaedic and other conditions. Orthopedic nursing. 2005;24(4):259-69.

20. Sibbritt D, Adams J, van der Riet P. The prevalence and characteristics of young and mid-age women who use yoga and meditation: results of a nationally representative survey of 19,209 Australian women. Complementary therapies in medicine. 2011;19(2):71-7.

21. Rao A, Sibbritt D, Phillips JL, Hickman LD. Prayer or spiritual healing as adjuncts to conventional care: a cross sectional analysis of prevalence and characteristics of use among women. BMJ Open. 2015;5(6). 
22. Brown WJ, Bryson L, Byles JE, Dobson AJ, Lee C, Mishra G, et al. Women's Health Australia: Recruitment for a National Longitudinal Cohort Study. Women \& Health. 1999;28(1):23-40.

23. Brown W, Bryson L, Byles J, Dobson A, Manderson L, Schofield M, et al. Women's health Australia: establishment of the Australian longitudinal study on women's health. Journal of Women's Health. 1996;5(5):467-72.

24. Women's Health Australia. Methods 2014c [cited 201416 June]. Available from: http://www.alswh.org.au/about/methods.

25. Women's Health Australia. Sample 2014b [cited 201416 June]. Available from: http://www.alswh.org.au/about/sample.

26. Ware JE, Kosinski M, Dewey JE, Gandek B. SF-36 health survey: manual and interpretation guide. Massacheusetts: Quality Metric Inc.; 2000.

27. Hosmer Jr DW, Lemeshow S. Applied Logisitic Regression. New York: John Wiley \& Sons; 2000.

28. Bell R, Suerken C, Quandt S, Grzywacz J, Lang W, Arcury T. Prayer for Health Among U.S. Adults: The 2002 National Health Interview Survey. Complementary Health Practice Review. 2005;10(3):175-88.

29. Honda K, Jacobson JS. Use of complementary and alternative medicine among United States adults: the influences of personality, coping strategies, and social support. Preventive Medicine. 2005;40(1):46-53.

30. Beckie TM. Biopsychosocial Determinants of Health and Quality of life Among Young Women with Coronary Heart Disease. Current Cardiovascular Risk Reports. 2013;8(1):1-10.

31. Lavie CJ, Milani RV. Adverse psychological and coronary risk profiles in young patients with coronary artery disease and benefits of formal cardiac rehabilitation. Arch Intern Med. 2006;166(17):1878-83.

32. Hollywell C, Walker J. Private prayer as a suitable intervention for hospitalised patients: a critical review of the literature. Journal of Clinical Nursing. 2009;18(5):637-51.

33. Hann D, Baker F, Denniston M, Entrekin N. Long-term Breast Cancer Survivors Use of Complementary Therapies: Perceived Impact on Recovery and Prevention of Recurrence. Integr Cancer Therapies. 2005;4(1):14-20.

34. Masters KS, Spielmans GI. Prayer and health: Review, meta-analysis, and research agenda. Journal of behavioral medicine. 2007;30(4):329-38.

35. Conti JM. The effects of intercessory prayer and transpersonal positive visualization on a hemodialysis population. Amherst: University of Massachusetts; 1999.

36. Bearon LB, Koenig HG. Religious cognitions and use of prayer in health and illness. The Gerontologist. 1990;30(2):249-53.

37. Stefano GB, Fricchione GL, Slingsby BT, Benson $\mathrm{H}$. The placebo effect and relaxation response: neural processes and their coupling to constitutive nitric oxide. Brain Research Reviews. 2001;35:1-19.

38. Das I, Anand H. Effect of Prayer and OM Meditation in Enhancing Galvanic Skin Response. Psychological Thought. 2012;5(2):141-9.

39. Koenig HG. Religion, Spirituality, and Health: The Research and Clinical Implications. ISRN Psychiatry. 2012;2012:33.

40. Pert CB. Molecules of emotion: Why you feel the way you feel. New York: Scribner; 1997.

41. Benor DJ. Spiritual healing: A unifying influence in complementary therapies.

Complementary Therapies in Medicine. 1995;3(4):234-8.

42. Schlitz M, Amorok T, Micozzi M. Consciousness and healing: Integral approaches to mindbody medicine. St Louis: Elseveir Churchill; 2005.

43. Wagner EH, Austin BT, Davis $\mathrm{C}$, Hindmarsh M, Schaefer J, Bonomi A. Improving chronic illness care: translating evidence into action. Health affairs. 2001;20(6):64-78. 